\title{
Further improvements in the sea urchin embryo cryopreservation protocol (Paracentrotus lividus)
}

\author{
Estefanía Paredes, Juan Bellas
}

Previous studies indicated the existence of some amount of variability in the embryonic development success of cryopreserved sea-urchin (Paracentrotus lividus) embryos. The present work studied the covariation of cryopreservation results fluctuations and reproductive parameters (condition index, $\mathrm{CI}$ and oocytes quality) during the reproductive season, in order to verify the hypothesis of the effect of sea-urchin energy allocated to reproduction on cryopreservation results on variability. Some methodological issues were also addressed, such as: the use of MiliQ water or Artificial Sea Water (ASW) for preparation of cryoprotectant solutions (CPAs); the determination of the optimum density of embryos, and the suitability of two different containers for cryopreservation ( $2 \mathrm{~mL}$ cryovials and $0.25 \mathrm{~mL}$ straws) and the possible differences in the cryopreservation outcome. The natural spawning period can be located when the condition Index decreases, at the beginning of summer. There is a positive correlation between the results obtained for the CI calculation among males and females $(\mathrm{r}=$ 0.63; $\mathrm{p}=0.02 ; \mathrm{n}=13$ ). There was also a positive correlation between female CI and the oocytes weight but, in both cases, no correlation was found between the cryopreservation outcome and either the CI of the female sea urchins or their oocyte weight. Embryos cryopreserved with CPAs prepared with ASW yielded $68 \pm 23 \%$ (average \pm standard deviation) growth after $96 \mathrm{~h}$ incubation, but when using CPAs prepared in MiliQ water, embryonic development was impaired and most blastulas were partially destroyed or lysed. Cryopreservation outcome achieved after $96 \mathrm{~h}$ incubation was not affected by the embryo density (10000; 5000; 2500; 1250; 500 larvae/vial), and the average larval growth was ca. $50 \pm 16 \%$ of the control larvae. No significant differences were found when using $0.25 \mathrm{~mL}$ straws or $2 \mathrm{~mL}$ cryovials, the average growth was $62 \pm 12 \%$ of the control larvae. In conclusion, the variability in cryopreservation results is not due to seasonality in sea-urchins reproductive condition and might be attributed to experimental variability. Once the reproductive season starts, we only need to check for healthy blastulas previous to cryopreservation, in order to achieve at least 50\% larval growth. Finally, although both straws and cryovials may be used indistinctly, it is advisable to use ASW for preparing CPA. 